\title{
048 PREDICTING PRODUCTIVITY OF THE HORIZONTAL WELL INSIDE LIMITED RESERVOIRS
}

AKIFI. IBRAGIMOV, MARINA N. BAGANOVA and ALEXANDER A. NEKRASOV Russian Academy of Sciences, Oil and Gas Research Institute, 63/2 Leninsky Prospect GSP-1, 117917 Moscow, Russia

\begin{abstract}
In the present paper new flow rate formulas for horizontal and slanting wells in isotropic and anisotropic reservoirs are presented. These formulas accurately approximate dependencies of the well productivity on geometric and hydrodynamic parameters of the system (creservoir + well), such as length, radius, and slanting angle of the well, distance to the external reservoir boundary, thickness of the layer, permeability, and anisotropy degree of the reservoir.

The impact of different parameters on the well performance has been studied with the use of highly accurate 3-D simulator. The developed analytical simulator is based on the modern theory of Newtonian and Heat potentials and on the domain decomposition principle, reducing the drainage problem in domains with complicated structure to problems in standard domains.

In case of a strictly horizontal well comparison between computational results and the constructed flow rate formula, as well as Borisov's, Pilatovsky's, Merkulov's, and Joshi's formulas is carried out. Qualitative and quantitative advantages of new formulas over the wellknown formulas is shown. These formulas may be used in other general computational finite elements and finite difference methods for modeling horizontal and slanting wells.

The results for both stationary and non-stationary cases may be used in inverse problem solution for defining hydrodynamic parameters of the layer.
\end{abstract}

\section{Introduction}

The fluids drainage induced by horizontal wells (HW) and slanting wells (SW) is still less fully studied than in the case of vertical wells (VW). The reason lies in the essentially 3-D character of fluid flow in the neighbourhood of HW or SW located in the middle of a limited reservoir, while the problem for VW may be considered as 2-dimensional. Analytical solution of the fluid flow problem subject to Darcy's law in 3-D statement is difficult to obtain.

At the same time, a number of analytical formulas (see $[1,2]$ ) linking production rate to differential pressure (productivity index) in relation to geometric and hydrodynamic parameters of the system (reservoir $+H W »$, valid under certain a priori conditions on the flow in the vicinity of HW and in the infinity, is available. These formulas are yet in rather poor agreement with the following classical and exact results: Dupuit's formula for perfect well, Musket's formula for imperfect well, and Adam's formula for a well located in an unbounded reservoir [3]. Further still, all the cited flow rate (FR) formulas refer to the case of strictly horizontal wells located in the middle of an isotropic reservoir.

The absence of accurate formulas, relating productivity index to the geometry of the system «reservoir + HW», poses a limit on accurate prediction of the HW performance and doesn't allow to solve inverse problems.

The main target of the present work consists in construction of new FR formulas for $\mathrm{HW}$ and SW in isotropic and anisotropic reservoirs accurately accounting for the qualitative and quantitative impact of geometric and hydrodynamic parameters on the well productivity.

The analytical simulator applied for this purpose is based on: 
-presentation of pressure function in the discrete form of Newtonian and Heat potentials with unknown density distribution concentrated on the axis of the well;

-iterative algorithms based on the decomposition principle reducing the initial problem in a complex domain to a sequence of problems in standard or simple domains such as sphere, cylinder, half-space, etc.

In [4] high accuracy $(3-7 \%)$ of the proposed approach is proved.

The developed analytical simulator served as a tool for a series of computational experiments, where well length, radius, slanting angle, thickness of the layer, and distance to the external reservoir boundary were varied. Analysis of the obtained numerical dependencies and application of important potentials properties produced new FR formulas presented in this paper.

The dependencies obtained from the simulator runs, the new FR formulas, and the previously known formulas (in those cases when they exist) are illustrated on figures 2-3 and may be compared. Note that for thick and isotropic layers these results were given in [1].

\section{Statement of the problem and solution method in stationary case}

In this section the stationary drainage problem for one-phase incompressible fluid. subject to anisotropic Darcy's law is considered.

$$
\vec{w}=-\frac{k}{\mu} \cdot \operatorname{grad} P, \quad \Rightarrow \quad L P=\sum_{i=1}^{3} \frac{\partial}{\partial x_{i}}\left(k_{i}(x) \sum_{i=1}^{3} \frac{\partial P}{\partial x_{i}}\right)=0
$$

$k_{1}=k_{2}=k_{b}$ and $k_{3}=k_{v}$ are permeabilities in horizontal and vertical directions correspondingly.

The flow region may be represented schematically by a 3-D spherical layer with a cylinder W (well) cut from it (see Fig. 1). At the top $\left(x_{3}=h\right)$ and bottom $\left(x_{3}=0\right)$ of the reservoir no-flow conditions are given. The pressure function is specified on the external reservoir boundary $S$ $\left(0 ; R_{b}\right)$ and on the $W$. Finally, the problem for the pressure function $P(x)$ takes the form of mixed boundary Zaremba's problem for elliptic operator with constant coefficients (see [4]).

By applying the theory of Newton's potential, the pressure function $P(x)$ can be represented in the following discrete form (see Fig. 2):

$$
P=\int_{W} G(x, \xi) \beta(\xi) d \xi \approx \sum \beta \int_{\Delta_{i}} G(x, \xi) d l_{\xi}
$$

where the unknown discrete densities distributions are found from the equation

$$
P(x,)=P \quad \text { when } x_{j} \in \partial W
$$

and $G(x, \xi)$ is the Green function of the problem

$$
\begin{aligned}
& \Delta G(x, \xi)=0 \text { in } \Omega \backslash \xi ; \\
& \frac{\partial G}{\partial x_{3}}=0 \text { when } x_{3}=0 \text { and } x_{3}=h ; \quad G=0 \text { on } S\left(0, R_{b}\right)
\end{aligned}
$$

In an infinite reservoir of finite thickness $\left(R_{b}=\infty, h=1 / 2\right)$ the function $G(x, \xi)$ is a superposition of infinite series of Green functions (see [3]). These series are, generally speaking, diverging, and they are transformed in [3] by means of specific asymptotic decompositions. This method is not applicable in case of a bounded reservoir $\left(R_{b}<\infty\right)$, consequently, an iterative method for construction of this type of Green functions was developed in [4]. The essence of this method consists in building up special function, defined in a ball (not only in the layer), Lharmonic everywhere except the point $\xi$, and such, that its restriction to the flow region satisfies the boundary conditions.

Flow Rate Formula. Applying the described method, one can obtain dependencies of the HW flow rate on the geometry of the system «reservoir $+H W$ s. Analysis of these dependencies produced a new flow rate formula, which proofed to be highly precise for productivity evaluation of a HW located in the middle of a bounded 3-D reservoir. 


$$
\begin{gathered}
Q=\frac{k}{\mu}\left[A_{1}\left(L, R_{k}\right) \cdot A_{2}\left(R_{k}, h\right) \cdot A_{3}(L, h) \frac{L}{\ln l / r_{*}}\right] \Delta P, \text { where } \\
A_{1}\left(L, R_{k}\right)=2 \pi+0.15 \frac{L}{R_{k}-L}, \quad A_{2}=\frac{0.5}{r_{*} / h \ln \frac{R_{k}}{h}+0.5}, A_{3}=\frac{0.85}{(L / h)^{0.6}+0.85}
\end{gathered}
$$

For the case of the well length commensurable to the reservoir thickness the FR formula is given in [1]. Comparison between computational results and analytical formulas is presented in Figs. 3, 4 (see graphics in [1] as well). On Fig. 3 and 4 the dependencies of productivity index (PI) on the well length and on the layer thickness are given.

Interference of Several HWs. Contrary to VWs, reduction of HWs interference problem to straightforward superposition of flows, even in case of two HWs, will result in significant mistakes. This can be accounted for by the fact, that the HW length is commensurable to and, in some cases, greater than the distance between them.

To solve the HWs interference problem the presented above method, as well as the classical Schwartz's method [5] have been applied. The essence of Schwartz's method consists in solving on each step of the iterative algorithm the problem for a single well with boundary conditions accounting for the changes in pressure function induced by other wells. The convergence degree of the developed algorithm is very high.

The computational results for two HWs are illustrated on Figs. 5 and 6 for the following cases:

a) the wells are parallel to the reservoir top and bottom and are located in the same vertical plane;

b) the wells are located in the same vertical plane at an angle $\alpha$,

c) the wells are parallel to each other and are located at the same distance from the reservoir

It can be observed in Fig. 6 that maximum flow rate in the case a) corresponds, as might be expected, to the distance between the wells equal $h / 2$.

As Fig. 5 indicates, maximum production rate in the case b) is attained at $\alpha=180^{\circ}$. The corresponding analytical formulas are given in [1].

Optimization of the SW parameters. The aim of this subsection is the study of the influence of the SW's slant angle and length on its productivity and optimization of these parameters.

The reservoir is schematically represented by spherical layer $\Omega$ and the well $W$ is a cylinder of radius $r_{W}$ and length $L$ its axes being situated at an angle $\alpha$ to the vertical axes $x_{3}$.

It is evident that the neighborhood of the reservoir top and bottom exerts primary effect on the performance of the SW. Thus, the source located at maximum distance from the no-flow barrier will have the highest productivity. This considerations and analysis of curve 1 on Fig. 7 allowed to obtain the following dependence of the SW productivity on the slant angle $\alpha$ :

$$
\begin{gathered}
Q(\alpha)=C \cdot F(\alpha)+Q_{H W}\left(L_{-x}, 2 H\right) L(\alpha) /\left(2 \cdot L_{\max }\right) \\
C=\left(Q_{r w}-Q_{H W}\left(L_{\max }, 2 H\right) \cdot H / 2 L_{\text {max }}\right) / F(0)
\end{gathered}
$$

where $Q_{V W}$ is the productivity of a perfect VW, $Q_{H W}\left(L_{m a x}, 2 H\right)$ is the productivity of a HW of length $L_{\max }$ located in the middle of the reservoir of thickness $2 H$.

When the well length is chosen subject to condition $L(\alpha)=\min \left\{H / \cos \alpha ; L_{\max }\right\}$, where $L_{\max }$ is the length limited by technological restrictions, analytical representation of $F(\alpha)$ is given in [1].

Computational results, obtained from this formula, are illustrated on Fig. 7. The value of $\gamma=$ 0.25 was chosen on the basis of numerical data analysis. Deviations of the computational results from those calculated from (7) doesn't exceed 10 percent on the entire interval of $\alpha$ values. Maximum mistake falls on the interval $\arccos \left(H / L_{\max }\right)<\alpha<\arccos \left(0.5 H / L_{\text {max }}\right)$, in all other case it is less than 5 percent.

Anisotropic case. In this subsection the problem of stationary inflow toward HW in a bounded anisotropic reservoir is studied: 
In this case at the first step the problem in initial anisotropic domain is reduced to an isotropic domain. Under the applied transformation spherical layer transforms into elliptic layer and circular cylinder, simulating HW, transforms into elliptic cylinder. Certain theorems of the potential theory [6] allow to relate anisotropic capacity of the initial circular domain with isotropic capacity of elliptic domain. The resulting FR formula for anisotropic layer takes the form:

$$
Q_{a}\left(k_{h} / k_{v}\right)=\frac{2 \sqrt{k_{h} / k_{v}-1}}{\sqrt{k_{h} / k_{v}} \ln \left[\left(\sqrt{k_{h} / k_{v}}+\sqrt{k_{n} / k_{v}-1}\right) /\left(\sqrt{k_{h} / k_{v}}-\sqrt{k_{h} / k_{v}-1}\right)\right]}
$$

Two graphics presented in Fig. 8 allow to compare the results obtained from the simulator runs and the above formula for different degrees of anisotropy.

\section{Non-stationary case}

In this section the problem of non-stationary inflow toward HW or SW in a bounded reservoir is studied. The research is concerned with the effect of the geometry of the system «reservoir + HW» on the dynamics of the productivity coefficient.

As in the stationary case, 3-D analytical simulator based on parabolic principle of domain decomposition and on the solutions representation in the form of heat potentials is constructed. The simulator accuracy is validated on test problems [5]. The obtained computational results enabled to derive a non-stationary inflow rate formula taking into account the essentially 3-D nature of the flow.

The solution method. The proposed method is based on the idea of SF superposition for the mixed problem (see [5]). Let the sources be located on the well axis coinciding with the axis $\boldsymbol{x}_{\boldsymbol{l}}$. The time interval is represented by a discrete sequence and the solution at the moment $t_{k}$ is sought in the form:

$$
P=\sum_{k=0}^{k_{0}} \sum_{t=1}^{N} \beta_{i}^{*} \int_{\tau_{k-1}}^{*}\left(\int_{\Lambda_{i}} G\left(x-\xi_{i} ; t-\tau\right) d l_{\xi}\right) d \tau
$$

where the source function, designated from here on as the Green's function, satisfies the corresponding initial value boundary conditions. For the purpose of simplicity we assume $P_{0}=0$. The unknown coefficients are determined from the condition:

$$
\left.P\right|_{t=t_{k}}=P_{w} \text {, when } x=x_{i}^{M} \in \partial T \text {, }
$$

here $x_{i}^{M}$ is a sequence of points on the well boundary fixed at every given moment $t=t_{k}$. The solution at each time inteval is sought in the form of simple layer potential with unknown distribution function. The effect of preceding intervals is accounted for by appropriate disturbance of boundary conditions.

The aim of computational experiments is to define the effect of a) distance from the well to the no-flow barrier, b) reservoir thickness, and c) well length on the stabilization rate providing the drainage is elastic.

By means of the developed analytical simulator great number of computational experiments have been conducted aimed at acquisition of inflow rate formula for a HW located in a 3-D unlimited reservoir bounded only by top and bottom. The fluid inflow towards the wellbore is conditioned only by its elastic properties and the difference of the initial reservoir pressure and the well pressure. If the well is located in the middle of the reservoir parallel to the top and bottom, the dependence of flow rate on the pressure drop is fairly accurately described by the following formula:

$$
Q=\frac{k m}{\mu}\left(\pi \frac{L}{L / h+\ln (L / h)}+\sqrt{\pi} \frac{L}{\ln (L / h)} \frac{r_{r}}{\sqrt{t}}\right) \operatorname{erf}\left(\frac{h}{\sqrt{\chi^{I}}}\right)
$$

This formula can be used to advantage in hydrodynamic investigation of the system «reservoir + well». 
Conclusions

1. Taking into account the essentially $3 \mathrm{D}$ character of flow in the neighborhood of a HW results in significantly different qualitative and quantitative dependencies of productivity index on the geometry of the system «reservoir $+\mathrm{HW}$ ».

2. The developed simulators, based on analytical and computational methods and representing the solutions in the form of Newton's and heat potentials, proofed to be highly accurate and enabled to obtain new flow rate formulas.

3. The optimal slant angle of a SW depends essentially on the limitations on the wellbore length and is the same under stationary and non-stationary flow conditions.

4. The SW offers substantial advantages as compared to a perfect VW. At given technological constraints under same reservoir pressure the SW productivity is several times grearter than that of a perfect VW.

5. If the wellbore length is less than reservoir thickness the effect of the no-flow barrier on the well performance is virtually negligeble.

References

1. Ibragimov, A.I., Baganova, M.N., Nekrasov, A.A. "Stationary and Non-Stationary Flow of Fluid in a Limited Reservoir and Inside Horizontal Well", SPE 97-132, presented at 1997 The Petroleum Society Annual Technical Meeting, Calgary, June 8-11 1997.

2. Joshi S.D.: Horizontal Well Technology 1987, PenWell Publishig Companyl Tulsa 1991

3. Muskat, M.: The Flow of Homogeneous Fluids Through Porous Media, McGraw-Hill, 1937

4. Ibragimov, A.I., Necrasov, A.A.: "An Analogue of Schwartz's Method for Construction of the Green's Function for Zaremba's Problem", J. of Comp. Math. and Math. Physics, Moscow (12, 1997).

5. Baganova, M., Ibragimov, A.: "On One Alternating Method for the Solution of Non-Stationary Filtration Problem in Complex Domains", Computational Technologies, Novosibirsk, (vol.1, 1996) pp. 5-12.

6. Landkoff, N.S.: Foundations of Modern Potential Theory, Moscow, Nauka, 1966.

\section{Nomenclature}

$\mathrm{Q}=$ flow rate of single well; $\mathrm{Q}_{\Sigma}=$ total flow rate of several wells; $C I=\frac{\mu Q}{k \Delta P} \quad$ = productivity index; $\mu$

$=$ viscosity; $\mathrm{R}_{\mathrm{k}}=$ radius of the external reservoir boundary (no-flow barrier) $; \mathrm{r}_{\mathrm{w}}=$ well radius; $\mathrm{L}$ = well length; $\mathrm{h}$ = reservoir thickness; $\mathrm{S}\left(0, \mathrm{R}_{\mathrm{k}}\right)=$ boundary of the ball; $\xi=$ point on the axis of the well; $\Omega=$ region of the drainage; $\mathrm{k}=$ reservoir permeability; $\mathrm{P}=$ pressure; $\alpha=$ slant angle.

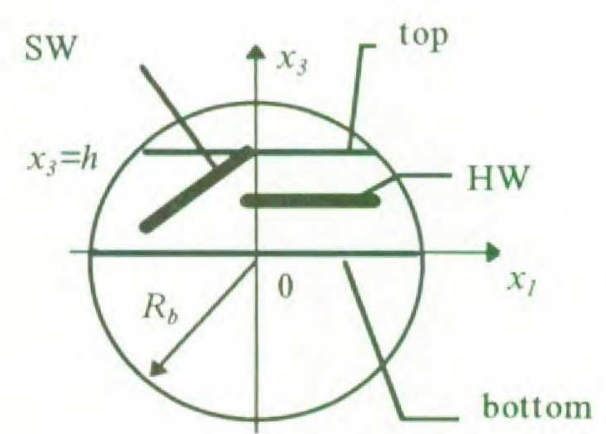

Fig. 1. Scheme of the reservoir

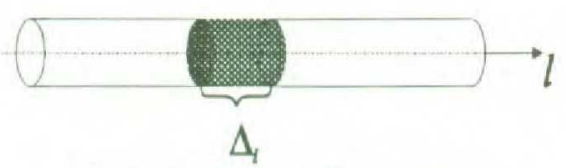

Fig. 2 The well model. 

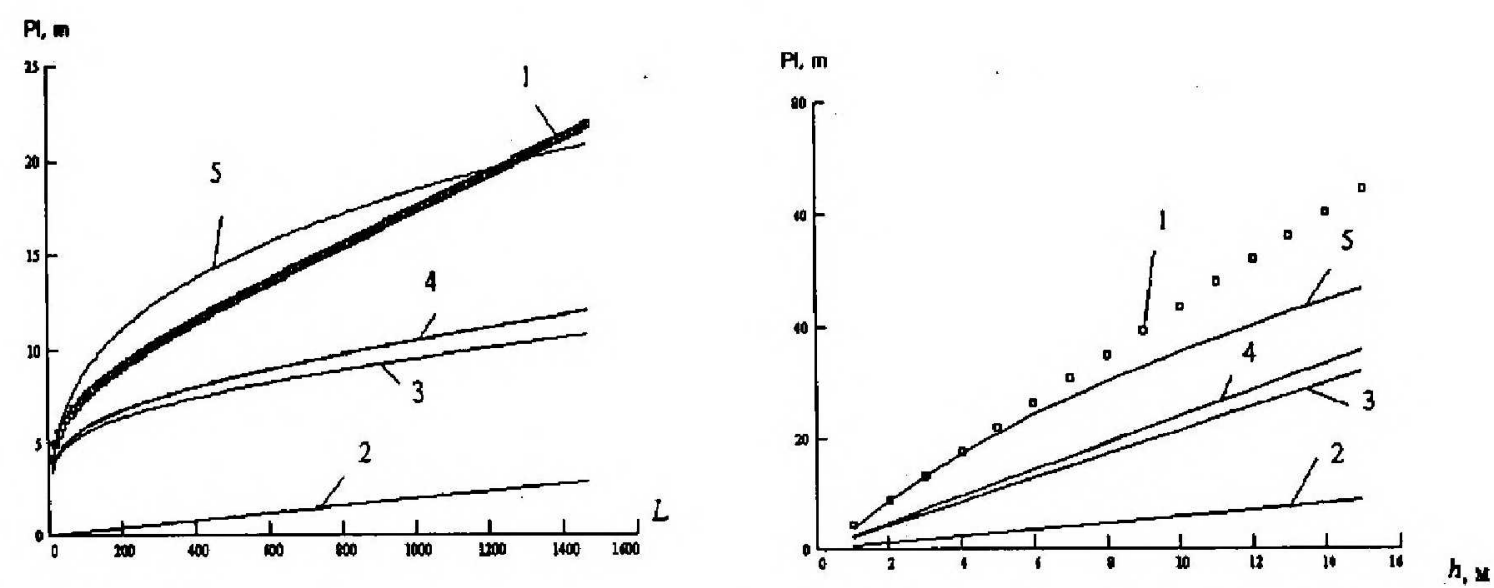

Fig.3. Productivity index vs. well length:

Fig.4. Productivity index vs. layer thickness 1 - simulator run; 2 - Merkulov's formula; 3 - Joshi's formula.4 - Pilatovsky's formula; 5 - formula (6);
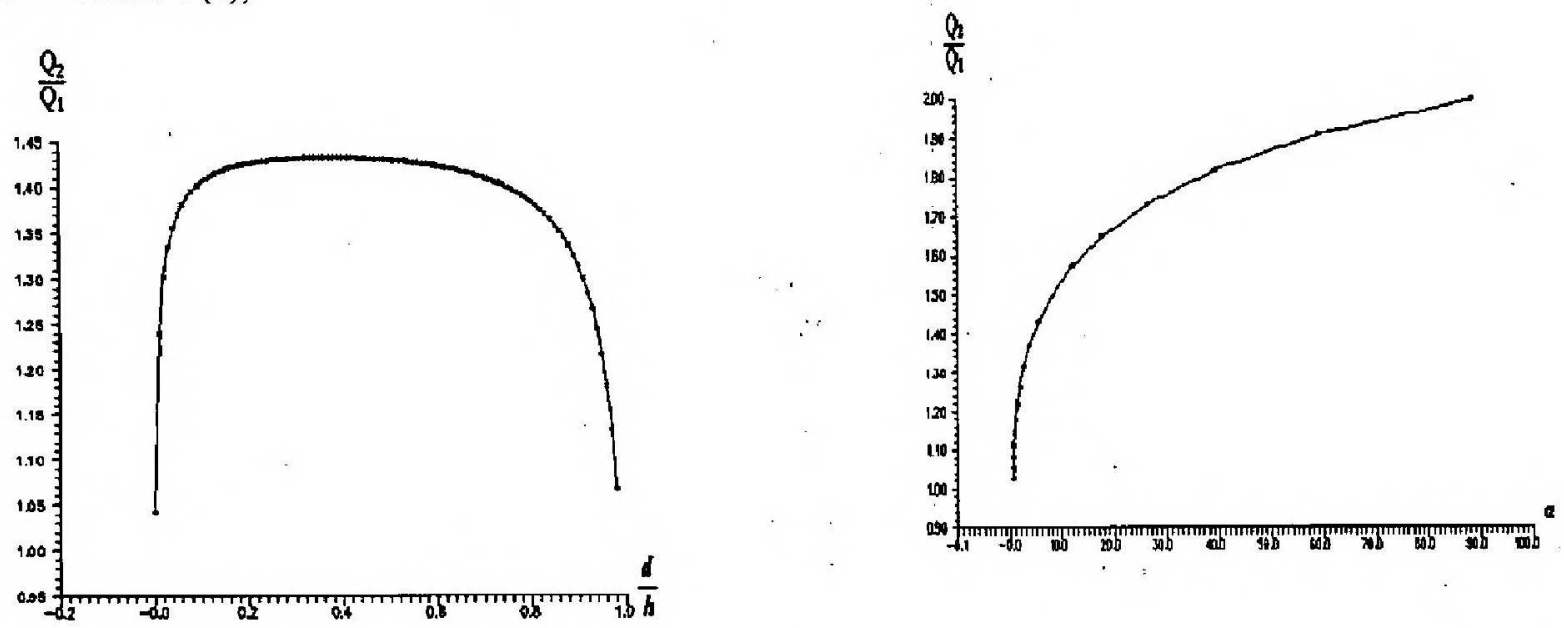

Fig. 5. Total productivity vs. distance between wells. wells

Fig. 6. Total productivity vs angle between
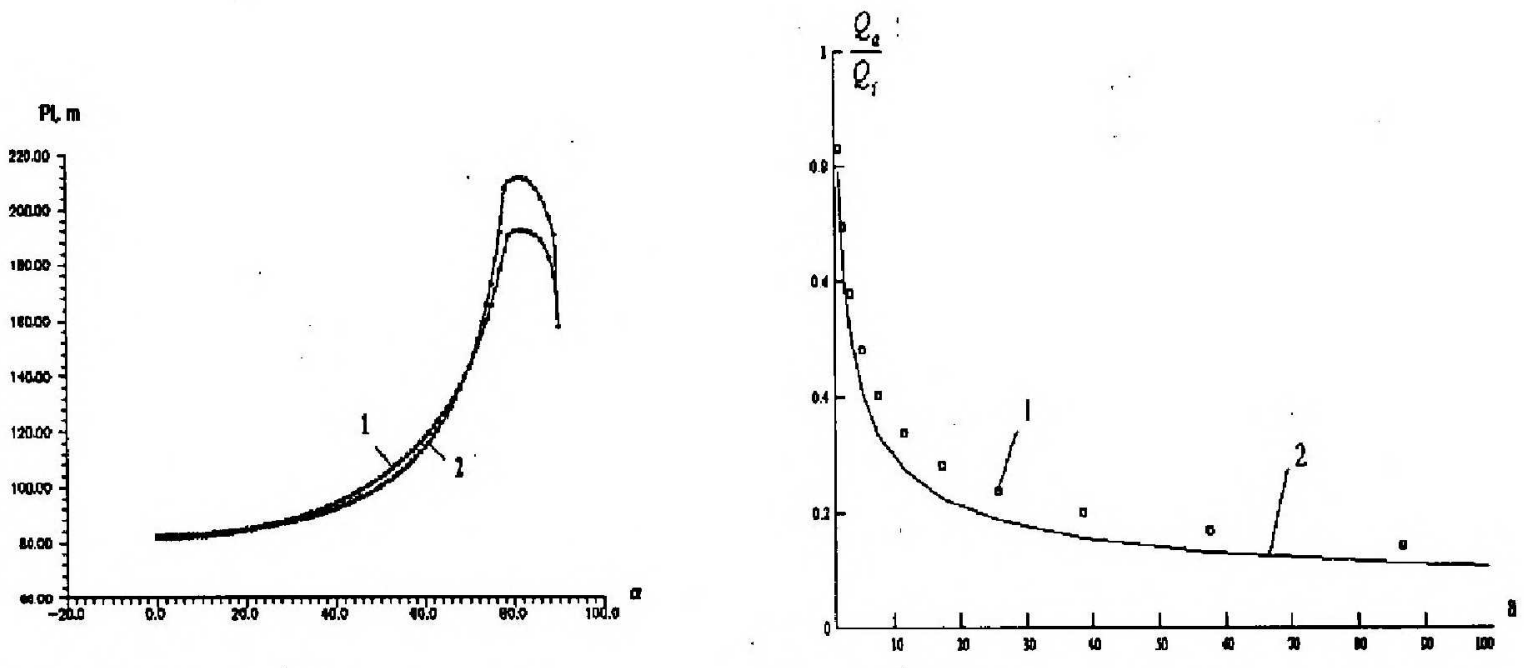

Fig. 7. SW productivity vs. angle $\alpha$.

.1 - simulator run; 2 - formula (7)

Fig. 8. HW Productivity Index vs. degree of anisotropy $\left(a=k_{v} / k_{k}\right)$ 